\title{
$W^{p}$-SPACES AND FOURIER TRANSFORM
}

\author{
R. S. PATHAK AND S. K. UPADHYAY \\ (Communicated by Palle E. T. Jorgensen)
}

\begin{abstract}
The spaces $W_{M}^{p}, W_{M, a}^{p}, W^{\Omega, p}, W^{\Omega, b, p}, W_{M}^{\Omega, p}, W_{M, a}^{\Omega, b, p}$ generalizing the spaces of type $W$ due to Gurevich (also given by Friedman, and Gelfand and Shilov) are investigated. Here $M, \Omega$ are certain continuous increasing convex functions, $a, b$ are positive constants and $1 \leq p<\infty$. The Fourier transformation $F$ is shown to be a continuous linear mapping as follows: $F: W_{M, a}^{p} \rightarrow W^{\Omega, 1 / a, r}, F: W^{\Omega, b, p} \rightarrow W_{M, 1 / b}^{r}, F: W_{M, a}^{\Omega, b, p} \rightarrow$ $W_{M, 1 / b}^{\Omega, 1 / a, r}$. These results will be used in investigating uniqueness classes of certain Cauchy problems in future work.
\end{abstract}

\section{THE SPACES $W_{M}^{p}, W_{M, a}^{p}$}

Let $\mu(\xi)$ be a continuous increasing function on $[0, \infty)$ such that $\mu(0)=0$, $\mu(\infty)=\infty$ for $x \geq 0$ define an increasing convex continuous function $M$ by

$$
M(x)=\int_{0}^{x} \mu(\xi) d \xi, \quad M(-x)=M(x) .
$$

Then $M(0)=0, M(\infty)=\infty$, and

$$
M\left(x_{1}\right)+M\left(x_{2}\right) \leq M\left(x_{2}+x_{2}\right) .
$$

Now the space $W_{M}^{p}(\mathbb{R})$ is defined as the set of all infinitely differentiable functions $\varphi(x) \quad(-\infty<x<\infty)$ satisfying

$$
\left(\int_{-\infty}^{+\infty}\left|e^{M(a x)} \varphi^{(q)}(x)\right|^{p} d x\right)^{1 / p} \leq C_{q, p}, \quad 1 \leq p<\infty,
$$

for each nonnegative integer $q$ where the positive constants $a$ and $C_{q, p}$ depend upon $\varphi$. Clearly $W_{M}^{p}$ is a linear space. The space $W_{M}^{p}$ can be regarded as the union of countably normed spaces $W_{M, a}^{p}$ of all complex valued $C^{\infty}$ functions $\varphi$, which for any $\delta>0$ satisfy

$$
\left(\int_{-\infty}^{+\infty}\left|e^{M[(a-\delta) x]} \varphi^{(q)}(x)\right|^{p} d x\right)^{1 / p} \leq C_{q, p}, \quad q=0,1,2, \ldots
$$

Received by the editors April 20, 1992 and, in revised form, October 6, 1992.

1991 Mathematics Subject Classification. Primary 46F12. 
The topology over $W_{M, a}^{p}$ is generated by

$$
\|\varphi\|_{p}=\sup _{q \leq l}\left(\int_{-\infty}^{+\infty}\left|M_{l}(x) \varphi^{(q)}(x)\right|^{p} d x\right)^{1 / p}
$$

where

$$
M_{l}(x)=\exp (M[a(1-1 / l) x]), \quad l=2,3, \ldots .
$$

From [2, p. 85] it follows that $W_{M, a}^{p}$ is sequentially complete countably normed linear space. A generalized function belonging to dual space $\left(W_{M, a}^{p}\right)^{\prime}, p \geq 1$, has the form

$$
f=\sum_{s=0}^{l} D^{s}\left[M_{l}(x) f_{s}(x)\right]
$$

for some nonnegative integer $l$, where the $f_{s}$ are measurable functions such that

$$
\sum_{s=0}^{l} \int_{-\infty}^{\infty}\left[\left|f_{s}(x)\right|^{q}\right] d x<\infty \quad\left(\frac{1}{p}+\frac{1}{q}=1\right) .
$$

For $a=0$, the space $W_{M, a}^{p}(\mathbb{R})$ reduces to the Schwartz space $D_{L} p(\mathbb{R})$.

The following properties can be established by using techniques similar to those employed in [2, pp. 12-13].

(1) The operation of differentiation is bounded in $W_{M}^{p}$ and is a continuous operation.

(2) The operation of multiplication by $x$ is bounded in $W_{M}^{p}$ and is a continuous operation.

\section{THE SPACES $W^{\Omega, b, p}, W^{\Omega, p}$}

Let $\Omega$ be another increasing, continuous, convex function possessing properties similar to $M$. Then $W^{\Omega, p}$ is defined to be the set of all entire analytic functions $\varphi(z) \quad(z=x+i y)$ satisfying the inequalities

$$
\left(\int_{-\infty}^{+\infty}\left|e^{-\Omega(b y)} z^{k} \varphi(z)\right|^{p} d x\right)^{1 / p} \leq C_{k, p}
$$

where the constants $C_{k, p}$ and $b$ depend on the function $\varphi$. A sequence $\left\{\varphi_{\nu}(z)\right\} \in W^{\Omega, p}$ is said to converge to zero if the functions $\varphi_{\nu}(z)$ converge to zero uniformly on any bounded domain of the $z$-plane and satisfy the inequalities

$$
\left(\int_{-\infty}^{+\infty}\left|e^{-\Omega(b y)} z^{k} \varphi_{\nu}(z)\right|^{p} d x\right)^{1 / p} \leq C_{k, p}, \quad k=0,1,2, \ldots,
$$

where the constants $C_{k, p}$ and $b$ do not depend on the index $\nu$. The space $W^{\Omega, b, p}$ defined below consists of the set of all those functions in $W^{\Omega, p}$ which satisfy the inequalities

$$
\left(\int_{-\infty}^{+\infty}\left|\exp \{-\Omega[(b+\rho) y]\} z^{k} \varphi(z)\right|^{p} d x\right)^{1 / p} \leq C_{k, p} .
$$


The topology over $W^{\Omega, b, p}$ is generated by the norms

$$
\|\varphi\|_{k, \rho, p}=\|\varphi\|_{k, \rho, b, p}=\left(\int_{-\infty}^{+\infty}\left|\exp \{-\Omega[(b+\rho) y]\} z^{k} \varphi(z)\right|^{p} d x\right)^{1 / p}
$$

with these norms $W^{\Omega, b, p}$ is a complete, perfect, countably normed linear space.

Theorem 2.1. Let $f(z)$ be an entire analytic function satisfying the inequalities

$$
\left\|\left(1+|x|^{h}\right)^{-1} f(z)\right\|_{p} \leq D_{p} e^{\Omega\left(b_{0} y\right)}
$$

and $\varphi(z) \in W^{\Omega, b, q}$. Then $\varphi(z) f(z) \in W^{\Omega, b \div b_{0}, r}, 1 / p+1 / q=1 / r$.

Proof. Since $\varphi(z) \in W^{\Omega, b, q}$ for $r>0$, we have

$$
\begin{aligned}
& \left(\int_{-\infty}^{+\infty}\left|\exp \left\{-\Omega\left[\left(b+b_{0}+\rho\right) y\right]\right\} z^{k} f(z) \varphi(z)\right|^{r} d x\right)^{1 / r} \\
& \leq\left(\int_{-\infty}^{+\infty}\left|\left(1+|x|^{h}\right) \exp \{-\Omega[(b+\rho) y]\} z^{k} \varphi(z)\right|^{q} d x\right)^{1 / q} \\
& \quad \times\left(\int_{-\infty}^{+\infty}\left|\frac{e^{-\Omega\left(b_{0} y\right)} f(z)}{1+|x|^{h}}\right|^{p} d x\right)^{1 / p} \\
& \leq D_{p}\left\{\left(\int_{-\infty}^{+\infty}\left|\exp \{-\Omega[(b+\rho) y]\} z^{k} \varphi(z)\right|^{q} d x\right)^{1 / q}\right. \\
& \left.\quad+\left(\left.\int_{-\infty}^{+\infty}|\exp \{-\Omega[(b+\rho) y]\}| x\right|^{h} z^{k} \varphi(z)^{q}\right)^{1 / q}\right\} \\
& \leq D_{p}\left\{C_{k, q}+C_{k, q+h}\right\} \leq D_{k, q} .
\end{aligned}
$$

Therefore $\varphi(z) f(z) \in W^{\Omega, b+b_{0}, r}$.

\section{THE SPACES $W_{M, a}^{\Omega, p}, W_{M}^{\Omega, p}$}

Let $M, \Omega$ be the same functions as defined in $\S 1$ and $\S 2$ respectively and let $a, b>0$. We denote by $W_{M}^{\Omega, p}$ the set of all entire analytic functions $\varphi(z)$ $(z=x+i y)$, which satisfy the inequalities

$$
\left(\int_{-\infty}^{+\infty}|\exp [M(a x)-\Omega(b y)] \varphi(z)|^{p} d x\right)^{1 / p} \leq C_{p}
$$

where the constants $a, b$, and $C_{p}$ depend upon the function $\varphi(z)$. Obviousiy the space $W_{M}^{\Omega, p}$ is a vector space over $\mathbb{C}$ under usual operations. A sequence $\left\{\varphi_{\nu}(z)\right\} \in W_{M}^{\Omega, p}$ is said to converge to zero if the function $\varphi_{\nu}(z)$ converges uniformly to zero in any bounded domain of the $z$-plane and in addition the following inequalities:

$$
\left(\int_{-\infty}^{+\infty}\left|\exp \{M(a x)-\Omega(b y)\} \varphi_{\nu}(z)\right|^{p} d x\right)^{1 / p} \leq C_{p}
$$

hold with constants $C_{p}, a, b$, which do not depend upon the index $\nu$. 
The space $W^{\Omega, p}$ can also be represented as a union of countably normed linear spaces. We denote by $W_{M, a}^{\Omega, b}, p$ the set of all those functions belonging to the space $W_{M}^{\Omega, p}$ which satisfy the inequalities

$$
\left(\int_{-\infty}^{+\infty}|\exp \{M[(a-\delta) x]-\Omega[(b+\rho) y]\} \varphi(z)|^{p} d x\right)^{1 / p} \leq C_{p}
$$

Define the following norms in the space $W_{M, a}^{\Omega, b, p}$ :

$$
\|\varphi\|_{\delta, \rho, p}=\left(\int_{-\infty}^{+\infty}|\exp \{M[(a-\delta) x]-\Omega[(b+\rho) y]\} \varphi(z)|^{p} d x\right)^{1 / p} .
$$

Example. Let us take $M(x)=x^{\alpha}, \Omega(y)=y^{\beta}, \alpha>1, \beta>1$. Then $W^{\Omega, p}$ consists of entire functions $\varphi(x+i y)$ satisfying

$$
\left\|\exp \left[a|x|^{\alpha}-b|y|^{\beta}\right] \varphi(x+i y)\right\|_{p} \leq C_{p}, \quad a, b, C_{p}>0 .
$$

Theorem 3.1. Let $f(z)$ be an entire analytic function satisfying the inequalities

$$
\left\|\exp \left[-M\left(a_{0} x\right)\right] f(z)\right\|_{p} \leq D_{p} \exp \left\{\Omega\left(b_{0} y\right)\right\}
$$

and $\varphi(z) \in W^{\Omega, b, q}$. Then $\varphi(z) f(z) \in W_{M, a-a_{0}}^{\Omega, b+b_{0}, r}, 1 / p+1 / q=1 / r$.

Proof. Let $\varphi(z) \in W_{M, a}^{\Omega, b, q}$ then for $r>0$ we have

$$
\begin{aligned}
& \left(\int_{-\infty}^{+\infty}\left|\exp \left\{M\left[\left(a-a_{0}-\delta\right) x\right]-\Omega\left[\left(b+b_{0}+\rho\right) y\right]\right\} f(z) \varphi(z)\right|^{r} d x\right)^{1 / r} \\
& \quad \leq\left(\int_{-\infty}^{+\infty}|\exp \{M[(a-\delta) x]-\Omega[(b+\rho) y]\} \varphi(z)|^{q} d x\right)^{1 / q} \\
& \quad \times\left(\int_{-\infty}^{+\infty}\left|\exp \left[-M\left(a_{0} x\right)-\Omega(b y)\right] f(z)\right|^{p} d x\right)^{1 / p} \\
& \quad<\infty
\end{aligned}
$$

Therefore, $f(z) \varphi(z) \in W_{M, a-a_{0}}^{\Omega, b+b_{0}, r}$.

\section{THE FOURIER TRANSFORMATION}

Theorem 4.1. Let $M(x), \Omega(y)$ be the pair of functions which are dual in the sense of Young. Then $F\left(W_{M, a}^{p}\right) \subset W^{\Omega, 1 / a, r}, p, r \geq 1$.

Proof. Let $\varphi \in W_{M, a}^{p}$. Then the Fourier transformation exists in the $L_{1}$ sense. Let $\psi$ be its Fourier transformation. Then by [2, pp. 21-22] is an entire 
function of $s=\sigma+i \tau$, and we have

$$
\begin{aligned}
& \left\|(i s)^{k} \psi(s)\right\|_{r}=\left(\int_{-\infty}^{+\infty}\left|(i s)^{k} \psi(s)\right|^{r} d \sigma\right)^{1 / r} \\
& =\left(\int_{-\infty}^{+\infty}\left(\frac{|s|^{k+2}+|s|^{k}}{\sigma^{2}+1}\right)^{r}|\psi(s)|^{r} d \sigma\right)^{1 / r} \\
& \leq\left(\int_{-\infty}^{+\infty}\left(\frac{|s|^{k+2}|\psi(s)|}{\sigma^{2}+1}\right)^{r} d \sigma\right)^{1 / r}+\left(\int_{-\infty}^{+\infty}\left(\frac{|s|^{k}|\psi(s)|}{\sigma^{2}+1}\right)^{r} d \sigma\right)^{1 / r} \\
& \leq\left(\int_{-\infty}^{+\infty} \frac{d \sigma}{\left(\sigma^{2}+1\right)^{r}}\left(\int_{-\infty}^{+\infty}\left|e^{-x \tau} \phi^{(k+2)}(x)\right| d x\right)^{r}\right)^{1 / r} \\
& +\left(\int_{-\infty}^{+\infty} \frac{d \sigma}{\left(\sigma^{2}+1\right)^{r}}\left(\int_{-\infty}^{+\infty}\left|e^{-x \tau} \phi^{(k)}(x)\right| d x\right)^{r}\right)^{1 / r} \\
& \leq\left(\int _ { - \infty } ^ { + \infty } \frac { d \sigma } { ( \sigma ^ { 2 } + 1 ) ^ { r } } \left(\left(\int_{-\infty}^{+\infty}\left|\exp M[(a-\delta) x] \varphi^{(k+2)}(x)\right|^{p} d x\right)^{1 / p}\right.\right. \\
& \left.\left.\times\left(\int_{-\infty}^{+\infty}\left|e^{x \tau} \exp -[M(a-\delta) x]\right|^{p^{\prime}} d x\right)^{1 / p^{\prime}}\right)^{r}\right)^{1 / r} \\
& +\left(\int _ { - \infty } ^ { + \infty } \frac { d \sigma } { ( \sigma ^ { 2 } + 1 ) ^ { r } } \left(\left(\int_{-\infty}^{+\infty}\left|\exp M[(a-\delta) x] \varphi^{(k)}(x)\right|^{p} d x\right)^{1 / p}\right.\right. \\
& \left.\left.\times\left(\int_{-\infty}^{+\infty}\left|e^{x \tau} \exp -[M(a-\delta) x]\right|^{p^{\prime}} d x\right)^{1 / p^{\prime}}\right)^{r}\right)^{1 / r} \\
& \leq C_{k+2, p}^{r} e^{\Omega(\tau / \gamma)}+C_{k, p}^{r} e^{\Omega(\tau / \gamma)} \\
& \left(\frac{1}{\gamma}=\left(\frac{1}{a}+\rho\right) \text { since } \gamma=a-2 \delta \text { and } \rho>0 \text { is arbitrarily small }\right) \\
& \leq D_{k, p}^{r} e^{\Omega[\tau(1 / a+\rho)]} \text {. }
\end{aligned}
$$

Theorem 4.2. Let $M(x)$ and $\Omega(y)$ be the same functions as in Theorem 4.1. Then

$$
F\left(W^{\Omega, b, p}\right) \subset W_{M, 1 / b}^{r}, \quad p, r \geq 1 .
$$

Proof. Since $\varphi(x+i y) \in W^{\Omega, b, p}$, it follows that

$$
\varphi(x+i y)=O\left(|x|^{-\delta}\right) \quad \text { as } x \rightarrow \infty
$$

for $\delta>0$ then following arguments given in [2, pp. 22-23] we obtain

$$
\left|\psi^{(q)}(\sigma)\right| \leq C_{q, \delta, p} e^{-\sigma y}\left(\left\|z^{q+2} \varphi(z)\right\|_{p}+\left\|z^{q} \varphi(z)\right\|_{p}\right) .
$$

Now using characteristic inequality and following the arguments [2, p. 23] we can estimate

$$
\left|\psi^{(q)}(\sigma)\right| \leq C_{q, \delta, p} \exp \left\{-M[(1 / b-\delta) \sigma]-M\left[\rho^{2} \sigma / b^{3}\right]\right\}
$$


Hence

$$
\left|\exp \{M[(1 / b-\delta) \sigma]\} \psi^{(q)}(\sigma)\right|^{r} \leq C_{q, \delta, p}^{r} e^{-r M\left(\rho^{2} \sigma / b^{3}\right)}
$$

so that

$$
\left\|\exp \{M[(1 / b-\delta) \sigma]\} \psi^{(q)}(\sigma)\right\|_{r} \leq D_{q, \delta, p}, r .
$$

From Theorems 4.1 and 4.2 we conclude that

Corollary. $F\left(W_{M, a}^{p}\right)=W^{\Omega, 1 / a, p}, F\left(W^{\Omega, b, p}\right)=W_{M, 1 / b}^{p}$.

Theorem 4.3. Let $\Omega_{1}(y)$ and $M_{1}(x)$ be the functions which are dual in the sense of Young to the functions $M(x)$ and $\Omega(y)$. Then

$$
F\left(W_{M, a}^{\Omega, b, p}\right) \subset W_{M_{1}, 1 / b}^{\Omega_{1}, 1 / a, r}, \quad p, r \geq 1 .
$$

Proof. Following the arguments given in [2, pp. 24-25] we can show that

$$
\begin{aligned}
|\psi(\sigma+i \tau)| \leq C_{\rho_{1}, \delta_{1}, p}^{\prime} \exp \left\{\left[-M_{1}[(1 / b-\delta) \sigma]\right.\right. & \left.-M_{1}\left\{\left(\rho^{2} \sigma / b^{3}\right)\right\}\right] \\
& \left.+\Omega_{1}\left[\left(1 / a+\rho_{1}\right) \tau\right]\right\} .
\end{aligned}
$$

Hence

$$
\begin{aligned}
\left|\exp \left\{M_{1}[(1 / b-\delta) \sigma]\right\} \psi(\sigma+i \tau)\right| & \\
& \leq C_{\rho_{1}, \delta, p}^{\prime} \exp \left\{-M_{1}\left(\rho^{2} \sigma / b^{3}\right)+\Omega_{1} \tau\left(1 / a+\rho_{1}\right)\right\}
\end{aligned}
$$

so that

$$
\left\|e^{M_{1}[(1 / b-\delta) \sigma]-\Omega_{1}\left[\left(1 / a+\rho_{1}\right) \tau\right]} \psi(\sigma+i \tau)\right\|_{r} \leq D_{\rho_{1}, \delta_{1}, p}^{1, r} .
$$

Corollary. $F\left[W_{M, a}^{\Omega, b, p}\right]=W_{M_{1}, 1 / b}^{\Omega_{1}, 1 / a, p}$

\section{REFERENCES}

1. A. Friedman, Generalised functions and partial differential equations, Prentice-Hall, Englewood Cliffs, NJ, 1963.

2. I. M. Gelfand and G. E. Shilov, Generalized functions, Vol. III, Academic Press, New York, 1967.

3. B. L. Gurevich, New types of test function spaces and spaces of generalized functions and the Cauchy problem for operator equations, dissertation, Kharkov, 1956. (Russian)

4. S. K. Upadhyay, On certain weighted $L^{p}$-spaces and Fourier and Hankel transforms of distributions, Ph.D. thesis, Banaras Hindu University, Varanesi, 1993.

Department of Mathematics, Banaras Hindu University, Varanasi-221005, INDia 